\title{
EDITORIAL \\ COVID-19 and its impact on neurosurgery: our early experience in Singapore
}

\author{
Zhiquan Damian Lee, MBBS, MRCS(Ed), David Low Chyi Yeu, MBBCh, FRCSEd(SN), \\ Beng Ti Ang, MBBS, FRCSEd(SN), Wai Hoe Ng, MD, FRACS, and Wan Tew Seow, MBBS, FRACS
}

Department of Neurosurgery, National Neuroscience Institute, Singapore

$\mathrm{T}$ HE novel coronavirus was first reported in Wuhan, China, in December 2019. Singapore's first confirmed COVID-19 case was reported on January 23, 2020, and from February 6th through 19th, Singapore had the highest number of cases outside of China. The World Health Organization (WHO) formally declared COVID-19 a pandemic on March 11, 2020.

For the past 17 years, Singapore had been preparing for such a pandemic since it experienced the Severe Acute Respiratory Syndrome (SARS) in 2003. Preparations for pandemic outbreaks have been a key part of the Singapore government and its healthcare institutions' business continuity planning and enterprise risk management since then.

Singapore is also fortunate in that the National Centre of Infectious Disease (NCID) was officially opened in September 2019. The NCID is a 330-bed, state-of-the-art, purpose-built facility designed to strengthen Singapore's capabilities in infectious disease management and prevention, housing clinical services (intensive care, quarantine and isolation facilitates, and operating rooms), the national infectious diseases laboratory, public health, research, training and education, and community engagement under one overarching structure. It became the operation center for the COVID-19 outbreak, with confirmed and suspected patients with COVID-19 initially admitted there and subsequently to the other public hospitals in Singapore.

Following the initial reports of the outbreak in China, precautionary measures were escalated to Disease Outbreak Response Condition (DORSCON) Yellow (DORSCON is a color-coded framework that shows the current disease situation, providing the public with general guidelines on what needs to be done to prevent and reduce the impact of infections: https://www.gov.sg/article/ what-do-the-different-dorscon-levels-mean). A govern- ment multiministry taskforce was assembled on January 22,2020 , to direct and coordinate the nation's efforts in response to the outbreak. The DORSCON alert level was raised to Orange on February 7, 2020, with the increasing number of confirmed cases and evidence of local community transmission emerging.

Singapore's public hospitals are grouped into 3 clusters, and each cluster has its own task force. Information and direction are received from the multiministry task force, and policies and management plans are then formulated by cluster task force and disseminated to their respective hospitals for execution. The National Neuroscience Institute (NNI), the largest neurosurgical service provider in Singapore, provides neurosurgical care to two-thirds of the country's population. It has multiple campuses present in 2 tertiary, 3 regional, and 1 pediatric hospitals. NNI's main campus is in the Tan Tock Seng Hospital where the NCID is also situated, and hence, the neurosurgical care delivery there is dependent on happenings at NCID.

In this short communication, we share our early experience with COVID-19 and illustrate its impact on the neurosurgery service and measures taken as part of Singapore's efforts against COVID-19.

\section{Equipping and Preparing Our Healthcare Workers}

Before the DORSCON alert level was raised to Orange, equipping healthcare workers was already underway. Each staff member had been fitted with 2 makes of N95 masks (in case there was a shortage of one type), and training on using powered air-purifying respirators (PAPRs) was organized. Eye shields and thermometers were issued. All healthcare workers had to report their temperatures twice daily and declare any sickness through a desktop or mobile-based staff health surveillance sys- 
tem. Temperature recording compliance is monitored and reminders issued to noncompliant staff.

\section{Neurosurgical Coverage Across Multiple Campuses}

Under DORSCON Orange, movement of consultants between hospitals was restricted. Manpower planning took into consideration subspecialty coverage (neurovascular, skull base, neurooncology) for tertiary hospitals, while the regional hospitals focused primarily on trauma and spine. However, depending on the urgency for surgery and the patient's stability for transfer, patients requiring specialized treatment would either be transferred to a tertiary hospital, or a consultant would be activated to perform the surgery in a regional hospital. This is reviewed on an individual case basis. Before interhospital transfer of patients, consultation with the infectious disease team and approval from the hospital's senior staff management is mandatory.

\section{Impact on Neurosurgery Service}

Elective surgeries are put on hold. Surgeries are reviewed by neurosurgeons and non-urgent cases are postponed. Semi-urgent cases can proceed, subject to the availability of operating theater staff and anesthetists. However, keeping in mind the unavoidable backlog of elective cases, non-urgent cases are reviewed weekly, and these surgeries are performed when possible. Cases not requiring a prolonged intensive care unit (ICU) stay are prioritized.

In Singapore, a 14-day isolation period was imposed on persons travelling from countries with COVID-19. If semi-urgent surgery was required in these patients, they were instead admitted in an isolation ward and underwent two COVID-19 swabs. When both tests returned as negative (within 48 hours), the patient was de-isolated and cleared for surgery. If urgent surgery were to be required for confirmed cases admitted to the NCID, it would be performed in the specially designed ORs there. However, thus far, this has not been necessary.

In the outpatient setting, specialist appointments were reduced to the minimum. Patients are screened 1 week before, and non-urgent appointments are postponed. Scans already performed are reviewed and phone consultation initiated with these patients. Patients receive medications at home via our pharmacy's home delivery service.

In solidarity with all healthcare workers, as the number of suspected cases increased, our neurosurgery staff, senior and junior alike, volunteered for 7-day rotations in the forefront screening centers to help screen suspected COVID-19 cases.

\section{Education and Meetings}

With restriction of interhospital movements, our department tapped into video conferencing for continuation of departmental Mortality and Morbidity Meetings, resident tutorials, and journal clubs. Physical distance is maintained by ensuring a group of fewer than 10 are present in the room each time.

\section{Maintaining Hygiene Practices and Morale}

The pandemic audit tool, encompassing personal protective equipment, hand hygiene, and environmental audits, is performed regularly to ensure consistency in hygiene practices. Glo Germ is used for environmental assessment and serves as a means of feedback to the housekeeping team. Compliance rates are reported to the NNI taskforce, and a weekly review is done. Peer support and welfare groups were formed to provide morale support for our staff - a priority - with helplines for easy access. Initiatives such "care packs" served as morale boosters, especially during trying times.

\section{Conclusions}

The fight against COVID-19 has clearly impacted our provision of neurosurgical service, yet the pandemic has only begun! We have learned important lessons from our early experience: First, the dynamic nature of this fight demands a clear line of information dissemination. Second, neurosurgeons share a duty in public health, and we need to work for the larger cause. Third, it is important for morale to be kept high. The race against COVID-19 will be prolonged, and resilience and team work are imperative in the coming days.

https://thejns.org/doi/abs/10.3171/2020.4.JNS201026

\section{Disclosures}

The authors report no conflict of interest.

\section{Correspondence}

Wan Tew Seow: seow.wan.tew@singhealth.com.sg.

INCLUDE WHEN CITING

Published online April 17, 2020; DOI: 10.3171/2020.4.JNS201026. 